\title{
MicroRNA-10a enhances the metastatic potential of cervical cancer cells by targeting phosphatase and tensin homologue
}

\author{
TIANHE ZENG ${ }^{1,2}$ and GUILING LI ${ }^{1}$ \\ ${ }^{1}$ Cancer Center, Union Hospital, Tongji Medical College, Huazhong University of Science and Technology, Wuhan, \\ Hubei 430023; ${ }^{2}$ The Affiliated Cancer Hospital of Zhengzhou University, Zhengzhou, Henan 450000, P.R. China
}

Received September 12, 2013; Accepted April 24, 2014

DOI: $10.3892 / \mathrm{mmr} .2014 .2370$

\begin{abstract}
Cervical cancer is one of the leading causes of cancer-related mortality worldwide. Previously, the upregulation of microRNA (miR)-10a has been identified in human cervical cancer. The present study firstly demonstrated that miR-10a was markedly upregulated in primary tumor tissues in patients with positive lymph node metastasis $\left(\mathrm{LN}_{+}\right)$compared with negative $\left(\mathrm{LN}_{\text {- }}\right.$ ) by quantitative polymerase chain reaction. miR-10a mimics markedly enhanced cervical cancer cell migration and invasion abilities, and accordingly the miR-10a inhibitor suppressed those functions. Furthermore, these data suggested that the phosphatase and tensin homologue (PTEN) was inhibited by miR-10a through an miR-10a binding site within the 3'-untranslated region of PTEN at the posttranscriptional level, and that miR-10a mimics promoted nuclear translocation of $\beta$-catenin. Therefore, it was concluded that the overexpression of miR-10a contributes to metastasis in cervical cancer by targeting PTEN. miR-10a may therefore be used clinically as a molecular marker for patients with cervical cancer lymph node metastasis.
\end{abstract}

\section{Introduction}

The presence of lymph node metastases is the main prognostic factor in early-stage cervical cancer patients. Although the International Federation of Gynecology and Obstetrics (FIGO) staging system for cervical carcinoma does not take into account the lymphadenopathy (LN) status, it is the most significant prognostic factor for patients with stages IB or IIA of the disease. In addition, the 5-year survival rate for patients declines markedly from $\sim 80-95 \%$ in patients without lymph node metastasis to $\sim 50-65 \%$ in patients with lymph node metastases (1). Radical hysterectomy and pelvic lymphadenectomy is the standard surgical treatment for early stage cervical

Correspondence to: Dr Guiling Li, Cancer Center, Union Hospital, Tongji Medical College, Huazhong University of Science and Technology, 156 Wujia Dun Road, Wuhan, Hubei 430023, P.R. China E-mail: 1g16714@163.com

Key words: microRNA-10a, cervical cancer, phosphatase and tensin homologue, metastasis, $\beta$-catenin nuclear translocation cancer. However, in a clinical study by Selman et al (2) it was confirmed that $84 \%$ patients did not exhibit pathology following a lymph node biopsy. Numerous studies have focused on strategies for detecting lymph node metastasis; however, the accuracy of these approaches, such as MRI and PET-CT, was identified to be only 50\% (3). Therefore, the identification of sensitive, accurate and non-invasive molecular markers may facilitate the selection of patients with lymph node negative cervical cancer with an unfavorable prognosis for adjuvant treatment and allow the identification of targets for patient-tailored therapy.

MicroRNA (miR) has an essential role in malignant tumor development and progression (4,5). Previous studies have demonstrated that certain miRs control tumor cell invasion and metastasis. For example, miR-200c initiated metastasis mediated by the loss of cell-cell adhesion caused by E-cadherin repression, in a process commonly termed epithelial-to-mesenchymal transition (EMT) (6). In addition, miR-26a enhanced the metastatic potential of lung cancer cells via the AKT pathway by targeting phosphatase and tensin homologue (PTEN) (7). Furthermore, enforced expression of miR-148a suppressed gastric cancer cell invasion and metastasis through directly targeting ROCK1 (8). The role of miR-10a in cancer metastasis has recently attracted notable attention. Microarray analysis data by Pereira et al (9) and Lee et al (10) demonstrated that miR-10a is upregulated in cervical cancer. It was also demonstrated that miR-10a triggered the metastatic properties of hepatocellular carcinoma by directly targeting EphA4 (11). Furthermore, the expression of miR-10a was at least 10-fold higher in three gastric cancer cell lines compared with gastric mucosal cell lines, and was involved in the development of gastric cancer and lymph node metastasis (12). In addition, circulating miR-10a was reported to be higher in the plasma of patients with non-small cell lung cancer compared with that in healthy volunteers (13). By contrast, controversial results have emerged reporting miR-10a to be a tumor suppressor by controlling cell migration/invasion in esophageal squamous cell carcinoma (14). However, the function of miR-10a in cervical cancer remains unknown and further study is warranted to elucidate this.

Therefore, the present study investigated the associated between the expression level of miR-10a and lymph node metastasis in cervical cancer, and further investigated the possible function of miR-10a in cervical cancer cell lines. The 
results revealed that the overexpression of miR-10a is significantly associated with metastasis and invasion in cervical cancer. Furthermore, it was able to negatively regulate PTEN by binding to the 3 '-untranslated region (UTR) regions to affect cervical cancer cell migration and invasion.

\section{Materials and methods}

Tissue samples and cell lines. Clinical data and pathological tissue samples were retrieved from the Cancer Center of Wuhan Union Hospital (Wuhan, China). The biopsy specimens from 40 unrelated patients who were diagnosed with FIGO IIb IIIb stage cervical cancer, were collected in 2012, and the pathology of these samples were confirmed as squamous cell carcinoma. None of the patients had previously received chemotherapy or radiotherapy. The present study was approved by the Institutional Review Board of the Wuhan Union Hospital. Written informed consent was obtained from all of the patients. Human tissues were frozen in liquid nitrogen and stored at $-80^{\circ} \mathrm{C}$. The Hela and Siha human cervical cancer cell lines were provided by the Gynaecology and Obstetric Laboratory of Wuhan Union Hospital (Wuhan, China). Hela cells were cultured in RPMI-1640 medium (Hyclone, Logan, UT, USA) complemented with $10 \%$ fetal bovine serum (FBS; Hyclone). The Siha cells were cultured in Dulbecco's modified Eagle's high glucose medium (Hyclone) complemented with $10 \%$ FBS (Hyclone). All of the cell lines were cultured in a humidified incubator $\left(5 \% \mathrm{CO}_{2}\right)$ at $37^{\circ} \mathrm{C}$.

Cell transfection. The miR-10a gain and loss-of-function studies were performed using miR-10a mimics (100 nM) and its negative control (mimic nc; $100 \mathrm{nM}$ ) and the loss-of-function study were performed with miR-10a inhibitor (150 nM) and its negative control (inhibitor nc; $150 \mathrm{nM}$ ) in Hela and Siha cell lines (all obtained from Guangzhou RiboBio Co., Ltd., Guangzhou, China). For each cell line, there was a blank control without any transfection. The cells were transfected using Lipofectamine ${ }^{\mathrm{TM}} 2000$ reagent (Invitrogen Life Technologies, Carlsbad, CA, USA) in Opti-minimal essential medium (Gibco-BRL, Carlsbad, CA, USA), according to the manufacturer's instructions. The relative expression level of miR-10a in transfected cells was examined by quantitative polymerase chain reaction (qPCR).

Dual-luciferase reporter assay. The region of human PTEN-3'UTR, generated by PCR amplification, was cloned into the pGL3 luciferase reporter plasmid. The primers selected were as follows: Forward: hsa-miR-10a 5'-CTCGAGGAAATAAAACCAAAGCACTC-3' and reverse: 5'-GGTACCGCCAGTCACCAGACTGTCCT-3' for hsa-miR-10a-R; and forward: 5'-TCTAGAAATGGC AATAGGACATTGTG-3' and reverse: 5'-TCTAGACCATCT TTATTAATCCTAATTG-3' for PTEN-3'UTR-wild type (wt). For the reporter assay, $239 \mathrm{~T}$ cells were plated onto 24-well plates and transfected with $500 \mathrm{ng}$ pGL3-miR-PTEN-wt using Lipofectamine 2000. Following transfection for $48 \mathrm{~h}$, the cells were harvested and the Renilla and Firefly luciferase were assayed by the Dual Luciferase Reporter Assay system (GeneChem, Shanghai, China) according to the manufacturer's instructions. The tests were repeated in triplicate.
$R N A$ extraction, reverse transcription and $q P C R$. Total RNA from the tissue samples and cells were isolated using TRIzol reagent (Ambion, Invitrogen Life Technologies). The relative expression levels of miR-10a were examined by the altered stem-loop RT-PCR with specific RT and PCR primers using U6 snRNA as a control. The primers for miR-10a were as follows: Forward: 5'-GCCGTACTC TGTAGATCCGAA-3' and reverse: 5'-CAGTGCAGGGTCCGAGGTAT-3'. The expression of PTEN mRNA was detected by qPCR using paired primers. The GAPDH gene was used as a control. The primers for PTEN mRNA were as follows: forward: 5'-AAGACCATAACCCACCACAGC-3' and reverse: 5'-GAGCCCCAGCCTTCTCCAT-3'. qPCR was performed on a Step One Plus Real-time PCR system (Applied Biosystems, Foster City, CA, USA) using SYBR Premix Ex $\operatorname{Taq}^{\mathrm{TM}}$ (Takara Biotechnology, Dalian, China) according to the manufacturer's instructions. The $2^{-\Delta \Delta \mathrm{Ct}}$ method, where $\Delta \Delta \mathrm{CT}=\left(\mathrm{Ct}\right.$ target gene-CtU6) $\mathrm{N}_{+}-(\mathrm{Ct}$ target gene-CtU6 $) \mathrm{N}_{0}$ was used for analysis. Where $\mathrm{N}_{+}$represents the patients positive for lymph node metastasis and $\mathrm{N}_{0}$ represents the patients negative for lymph node metastasis.

In vitro transwell invasion and migration assay. Transwell membranes (polycarbonic membrane; diameter, $6.5 \mathrm{~mm}$; pore size, $8 \mu \mathrm{m}$; Corning Costar, Inc., Corning, NY, USA) coated with Matrigel (BD BioSciences, USA) were used to assay the cell invasion and migration in vitro. At $48 \mathrm{~h}$ post-transfection, the cells were resuspended into serum-free medium. The transfected cells $\left(10 \times 10^{4}\right.$ in $200 \mu \mathrm{l}$ serum-free medium) were reseeded into the upper chamber, and $600 \mu 1$ medium with $10 \%$ FBS was added to the lower chamber as a chemoattractant. Following $24 \mathrm{~h}$ incubation, non-invading cells on the upper surface of the membrane were removed with a cotton swab. The invasive cells, which penetrated to the lower surface, were fixed with $4 \%$ paraformaldehyde and stained with $0.1 \%$ crystal violet. The number of cells invading the membrane was counted from five randomly selected visual fields with an inverted microscope (Olympus, Tokyo, Japan) at a magnification of x200. Data were obtained from three independent experiments.

Wound healing assay. The Hela and Siha cells were seeded onto 6 -well plates. When the cell confluence reached $\geq 80 \%$ and above at $\sim 48 \mathrm{~h}$ post-transfection, the scratch wounds were made by scraping the cell layer across each culture plate using the tip of $10 \mu \mathrm{l}$ pipette. Then five fields were randomly selected from each scratch wound and visualized by microscopy. The experiments were performed in triplicate.

Immunocytochemistry. The Hela and Siha cells were seeded onto 13 -mm glass coverslips on a 24 -well plate. At $48 \mathrm{~h}$ following transfection with the non-specific control, miR-10a mimic (100 $\mathrm{nM})$ cells were immunostained with anti- $\beta$-catenin antibody (Abnova, Taipei, Taiwan) according to the immunofluorescence staining instructions provided by the manufacturer. The labeled cells were observed with a A1Si confocal microscope (Nikon, Tokyo, Japan).

Western blotting. The Hela and Siha cells were harvested after $72 \mathrm{~h}$ following transfection with the miR-10a mimic, 

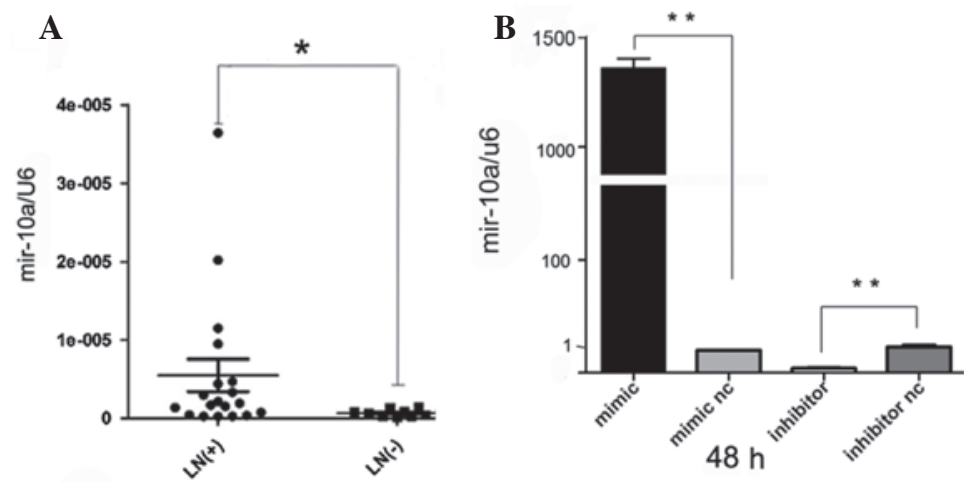

Figure 1. Level of miR-10a is upregulated in $\mathrm{LN}_{+}$tissues and following miR-10a mimic transfection. (A) RT-PCR analysis of the relative miR-10a expression levels in tumor tissues with or without pelvic lymph node metastasis $(\mathrm{P}<0.05)$. (B) Relative miR-10a expression in Hela cells $48 \mathrm{~h}$ following transfection with mimic, mimic nc, inhibitor and inhibitor nc. "P $\mathrm{P} 0.05,{ }^{* *} \mathrm{P}<0.01$ versus the control. miR, microRNA; $\mathrm{LN}_{+- \text {- }}$ positive/negative lymph node metastasis patients; nc, negative control.

A
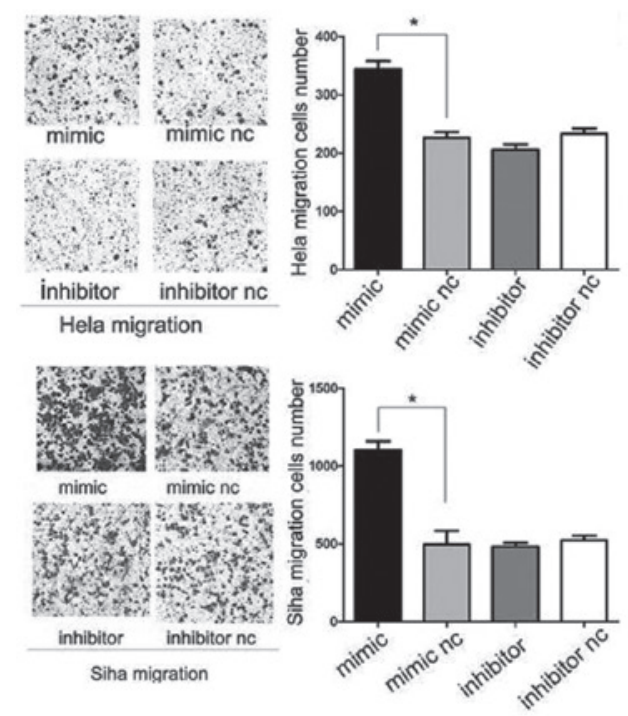

B

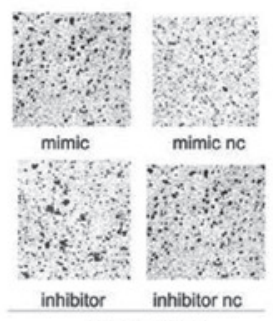
Hela invasion

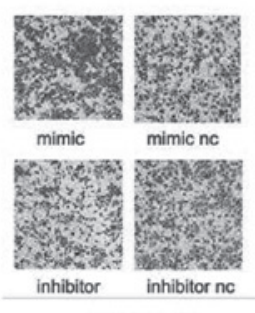

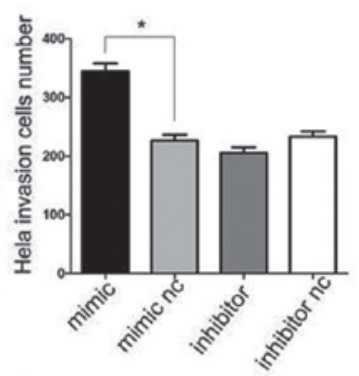

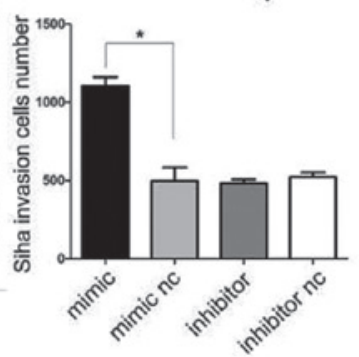

C

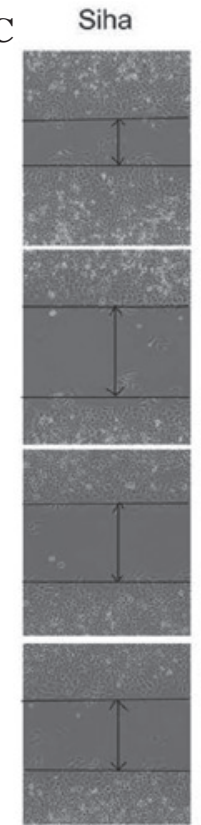

Hela
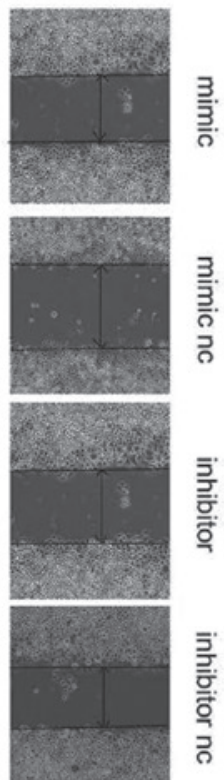

Figure 2. MicroRNA-10a enhanced the migration and invasion abilities of Siha and Hela cells. (A and B) Hela and Siha cells penetrating the membrane were fixed and $0.1 \%$ crystal violet stained following $24 \mathrm{~h}$ as described in Materials and methods ${ }^{*} \mathrm{P}<0.05,{ }^{* *} \mathrm{P}<0.01$ versus the control. (C) Migration ability was assessed by a wound healing assay following $72 \mathrm{~h}$ transfection (original magnification, $\mathrm{x} 200$ ). nc, negative control.

mimic nc, inhibitor or inhibitor nc. Equal quantities of the denatured protein sample were separated by SDS-PAGE and then transferred to polyvinylidene difluoride membranes (Wuhan Goodbio Technology Co., Ltd., Wuhan, China). The following primary antibodies were used: Anti-PTEN (dilution, 1:100; Abnova) and anti- $\beta$-actin (dilution, 1:400; Santa Cruz Biotechnology, Inc., Santa Cruz, CA, USA). Following overnight incubation at $4^{\circ} \mathrm{C}$ and washing, the membranes were probed with horseradish peroxidase-conjugated goat anti-rabbit antibody (Guge) at 1:5,000 dilution. The images were captured with the exposure machine (UVP OptiCam600; UVP Inc., Upland, CA, USA).

Statistical analysis. The results were analyzed using SPSS 17.0 software (SPSS, Inc., Chicago, IL, USA). Statistical analysis was performed using a Mann-Whitney test and one-way analysis of variance. $\mathrm{P}<0.05$ was considered to indicate a statistically significant difference.

\section{Results}

Expression level of miR-10a is upregulated in cervical cancer tissues with pelvic lymph node metastasis. To identify the miR-10a expression in the clinical samples, qPCR was performed and it was identified that following normalization to RNU6B expression levels, the expression level of miR-10a in cervical cancer tissues with $\mathrm{LN}_{+}$[mean \pm standard deviation (SD): $0.000003448+0.00008553$ ] was significantly higher than LN (mean \pm SD: $0.000001109+0.000002271 ; P=0.0053)$, as demonstrated in Fig. 1A. This data suggested that miR-10a may directly function in cervical cancer cell metastasis.

Upregulation of miR-10a expression promotes invasion and migration of cervical cancer cells. As the results above demonstrated that the upregulation of miR-10a in cervical cancer is closely associated with cervical cancer metastasis, it was hypothesized that the overexpression of miR-10a in cervical 
A

\section{3' guguuuaagccUAGAUGUCCCAu 5' has-mir-10a}

\section{5 : 5' uuacccuauacAUCCACAGGGUu 3' PTEN}

B
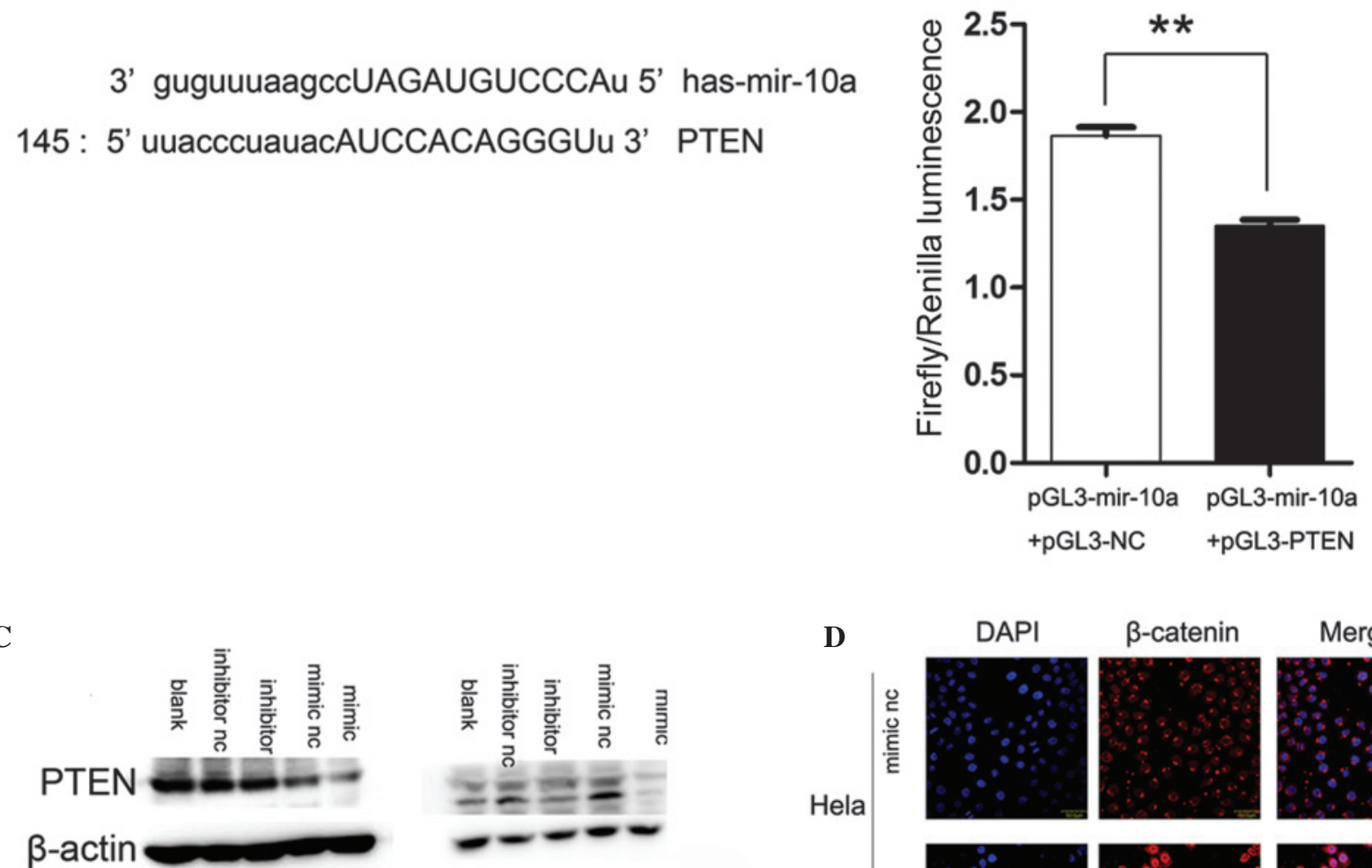

$\beta$-actin
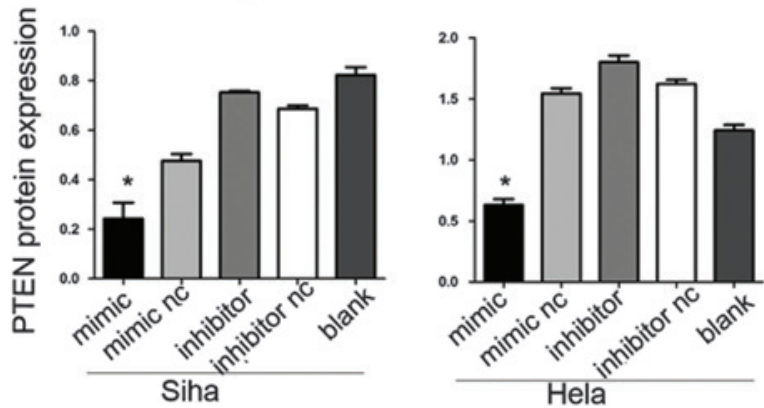

D
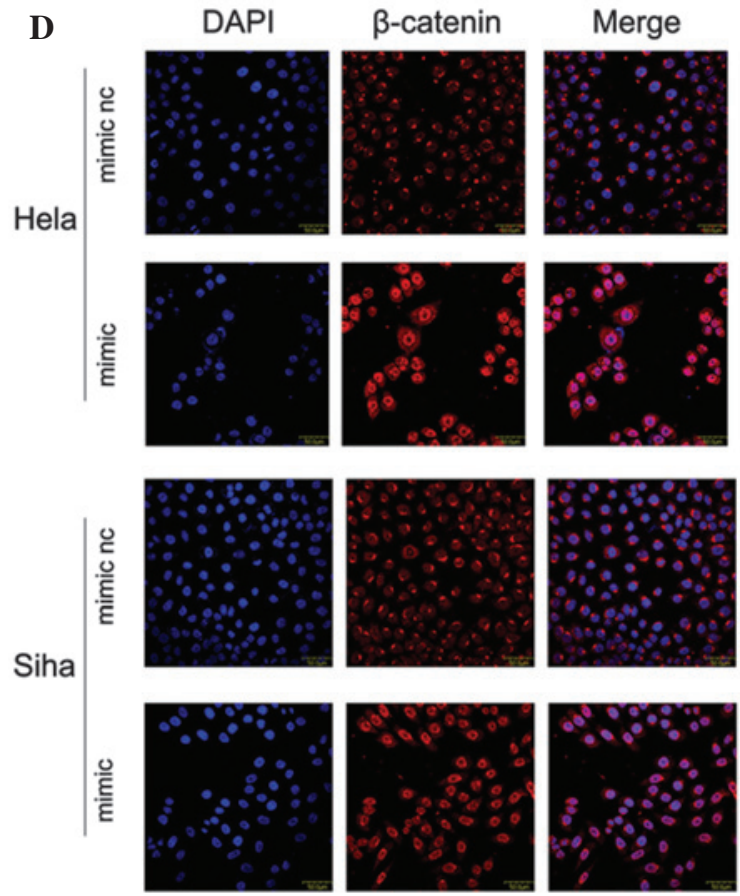

Figure 3. Mechanism of miR-10a-induced migration of cervical cancer cells. (A) Putative miR-10a binding sites in the 3'-UTR of PTEN. (B) Relative activity of the luciferase gene fused with the wt 3'-UTR of PTEN. The luciferase activities were measured following 24 h. (C) The expression levels of PTEN were detected by western blot analysis following $72 \mathrm{~h}$ transfection. ${ }^{*} \mathrm{P}<0.05,{ }^{* *} \mathrm{P}<0.01$ versus the control. (D) Immunofluorescence staining was performed at $72 \mathrm{~h}$ following the transfection with miR-10a mimic and mimic nc (100 nM). Scale bar, $50 \mu \mathrm{m}$. PTEN, phosphatase and tensin homologue; UTR, untranslated region; miR, microRNA; wt, wild type; nc, negative control.

cancer cells exerted effects of cell invasion and metastasis In gain-of-function and loss-of-function studies, Hela and Siha cells were separately transfected with mimic and mimic nc, inhibitor and inhibitor nc. Transfection with $100 \mathrm{nM}$ of miR-10a mimics in Hela cells led to an $\sim 1328$-fold increase in the miR-10a expression detected $48 \mathrm{~h}$ post-transfection, as examined by qPCR (Fig. 1B). It was identified that the overexpression of miR-10a induced Hela and Siha cell migration, while downregulated miR-10a could inhibit the migratory ability of Hela and Siha cells (Fig. 2A and C). Consistent with this finding, the matrigel invasion assay results demonstrated that miR-10a overexpression significantly enhanced the invasion capacity of Hela and Siha cells, while decrease of miR-10a could inhibit invasion in Hela and Siha cells, however, this was not statistically significant (Fig. 2B). These observations demonstrated that miR-10a had an important role in promoting migration and the invasive potential of cervical cancer cells.

miR-10a targets PTEN. The analysis conducted using publicly available programs, TargetScan (http://www.targetscan. org) and miRanda (http://www.microrna.org), indicated that PTEN is theoretically the target gene of miR-10a (Fig. 3A). Next, a luciferase reporter assay was performed to verify that miR-10a directly targets PTEN. It was identified that co-transfection of pGL3-miR-10a and pGL3-PTEN-wt, 
significantly decreased the luciferase activity in $239 \mathrm{~T}$ cells as compared with the control (Fig. 3B).

Alteration of miR-10a expression changes PTEN protein but not $m R N A$ expression levels. PTEN had been reported to be downregulated in cervical cancer and closely associated with lymph node metastasis. To further confirm that PTEN was the downstream target of miR-10a, upregulation and downregulation of miR-10a expression were conducted with subsequent detection of PTEN mRNA and protein changes. The increase in endogenous miR-10a levels significantly decreased PTEN protein expression as determined by western blot analysis $(\mathrm{P}<0.05$; Fig. 3C), while the mRNA expression of PTEN remained unchanged (data not shown).

miR-10a mimic promotes nuclear translocation of $\beta$-catenin. Considering that PTEN was able to restrain $\beta$-catenin nuclear translocation and miR-10a inhibited PTEN expression, it was determined whether miR-10a may affect the $\beta$-catenin nuclear translocation activity. Notably, the immunofluorescence staining assays demonstrated that the overexpression of miR-10a resulted in marked nuclear accumulation of $\beta$-catenin in Hela and Siha cervical cancer cell lines, suggesting that miR-10a may contribute to the accumulation of $\beta$-catenin in the nuclei (Fig. 3D).

\section{Discussion}

Lymph node status is one of the most important prognostic factors following cervical cancer diagnosis, which dominates the majority of clinical decisions regarding treatment $(15,16)$. As the current imaging techniques are not reliable to diagnose lymph node status, pelvic +/- para-aortic lymphadenectomy remains the gold standard. These surgical procedures are, however, responsible for specific morbidity: Lymphocele and lymphedema. However, lymph node metastasis is rarely identified by CT or MRI when they are $<1 \mathrm{~cm}$. Therefore, effective molecular markers for detecting lymph node metastasis are urgently required $(17,18)$. Previously, a number of miRNAs were identified to be lymph node metastasis biomarkers, including miRNA-10b and miRNA-373, which are used for detecting lymph node metastasis of breast cancer (19). Furthermore, miR-21, miR-212 and miR-195 were found to be promising novel biomarkers for lymph node metastasis in gastric cancer $(20,21)$, and it appears miR-20a may be a potential biomarker for detecting the lymph node status of cervical cancer patients (22). The miR-10 family containing five miRNA genes, including miR-10a, miR-10b, miR-196a-1, miR-196a-2 and miR-196b, has attracted notable attention due to its conservation and the position of the miR-10 genes within the Hox clusters of developmental regulators (23). For miR-10a, it interacts with the 5' untranslated region of mRNAs encoding RPs (ribosomal proteins) to enhance their translation. Accordingly, RPs are found to be deregulated in cancer (24). Furthermore, miR-10a was found overexpressed in glioblastoma, hepatocellular carcinomas, colon cancer and acute myeloid leukemia with NPM1 mutations (25-28). In the present study, it was identified that miR-10a in primary tumor tissue was markedly upregulated in $\mathrm{N}_{+}$patient samples. Next, the function and possible mechanisms of miR-10a in regu- lating certain biological properties of Hela and Siha cervical cancer cells was investigated. However, through MTT and flow cytometry, it was verified that miR-10a had no effect on the cell proliferation and apoptosis in $72 \mathrm{~h}$ (data not shown). The data also suggested that a miR-10a mimic triggered the migration and invasion capacities of cervical cancer cells by negatively regulating tumor suppressor PTEN at the transcriptional level via binding to non-coding regions of PTEN.

PTEN is a tumor suppressor that negatively regulates (mTOR)/PI3K/Akt pathways. PTEN is one of the most mutated and deleted tumor suppressors in human cancer and has an important role in tumor metastasis (29-32). Loss of PTEN is associated with lymph node metastasis of cervical cancer $(33,34)$, sentinel lymph node micro-metastasis in breast cancer (35) and cervical lymph node metastasis in salivary gland cancer (36). The present study found that an miR-10a mimic was able to inhibit PTEN at the transcriptional level. The function of PTEN is involved in the suppression of nuclear-catenin accumulation $(37,38)$. The results demonstrated that the miR-10a mimic was able to trigger $\beta$-catenin nuclear translocation. In cancer cells, $\beta$-catenin within the nucleus generates a positive and reinforcing feedback loop, resulting in the nuclear accumulation of $\beta$-catenin (39). A strong correlation has been identified between $\beta$-catenin nuclear translocation, tumor metastasis and poor prognosis (40-42). In cervical cancer, $\beta$-catenin nuclear translocation has been noted for its function in lymph node metastasis, growth, invasion and angiogenesis $(41,43)$. Stabilization and nuclear localization of $\beta$-catenin, which induces the expression of Wnt target genes and subsequently triggers EMT, may be associated with the induction of metastasis (44). The present study suggests that the mechanisms of miR-10a promoting the invasion and migration ability of Hela and Siha cells, may be that miR-10a inhibits PTEN protein expression and then promotes $\beta$-catenin accumulation in the nucleus. To the best of our knowledge, this mechanism has not been reported prior to this study and requires further investigation.

In conclusion, it was observed that miR-10a is overexpressed in cervical tumor tissues with lymph node metastasis, and miR-10a may significantly enhance the migration and invasion capacities of cervical cancer cells by inhibiting PTEN at the transcriptional level. To the best of our knowledge, these data have not been documented in previous studies. The present data suggests that miR-10a may be useful as a molecular marker for detecting patients with lymph node metastasis of cervical cancer. These findings warrant further studies with a large cohort of patients to validate and develop the tissue biomarker as a critical tool for cervical cancer care.

\section{References}

1. Sakuragi N: Up-to-date management of lymph node metastasis and the role of tailored lymphadenectomy in cervical cancer. Int J Clin Oncol 12: 165-175, 2007.

2. Selman TJ, Luesley DM, Murphy DJ and Mann CH: Is radical hysterectomy for early stage cervical cancer an outdated operation? BJOG 112: 363-365, 2005.

3. Monteil J, Maubon A, Leobon S, et al: Lymph node assessment with (18)F-FDG-PET and MRI in uterine cervical cancer. Anticancer Res 31: 3865-3871, 2011.

4. van Kouwenhove M, Kedde M and Agami R: MicroRNA regulation by RNA-binding proteins and its implications for cancer. Nat Rev Cancer 11: 644-656, 2011. 
5. Calin GA and Croce CM: MicroRNA signatures in human cancers. Nat Rev Cancer 6: 857-866, 2006.

6. Ceppi P, Mudduluru G, Kumarswamy R, Rapa I, Scagliotti GV, Papotti M and Allgayer H: Loss of miR-200c expression induces an aggressive, invasive, and chemoresistant phenotype in non-small cell lung cancer. Mol Cancer Res 8: 1207-1216, 2010.

7. Liu B, Wu X, Liu B, Wang C, Liu Y, Zhou Q and Xu K: MiR-26a enhances metastasis potential of lung cancer cells via AKT pathway by targeting PTEN. Biochim Biophys Acta 1822: 1692-1704, 2012.

8. Zheng B, Liang L, Wang C, et al: MicroRNA-148a suppresses tumor cell invasion and metastasis by downregulating ROCK1 in gastric cancer. Clin Cancer Res 17: 7574-7583, 2011.

9. Pereira PM, Marques JP, Soares AR, Carreto L and Santos MA MicroRNA expression variability in human cervical tissues. PLoS One 5: e11780, 2010

10. Lee JW, Choi CH, Choi JJ, et al: Altered microRNA expression in cervical carcinomas. Clin Cancer Res 14: 2535-2542, 2008.

11. Yan Y, Luo YC, Wan HY, et al: MicroRNA-10a is involved in the metastatic process by regulating Eph tyrosine kinase receptor A4-mediated epithelial-mesenchymal transition and adhesion in hepatoma cells. Hepatology 57: 667-677, 2013.

12. Chen W, Tang Z, Sun Y, et al: miRNA expression profile in primary gastric cancers and paired lymph node metastases indicates that miR-10a plays a role in metastasis from primary gastric cancer to lymph nodes. Exp Ther Med 3: 351-356, 2012.

13. Markou A, Sourvinou I, Vorkas PA, Yousef GM and Lianidou E: Clinical evaluation of microRNA expression profiling in non small cell lung cancer. Lung Cancer 81: 388-396, 2013.

14. Matsushima K, Isomoto H, Kohno S and Nakao K: MicroRNAs and esophageal squamous cell carcinoma. Digestion 82: 138-144, 2010.

15. Gouy S, Morice P, Narducci F, et al: Nodal-staging surgery for locally advanced cervical cancer in the era of PET. Lancet Oncol 13 e212-e220, 2012

16. Kato T, Watari $\mathrm{H}$, Takeda $\mathrm{M}$, et al: Multivariate prognostic analysis of adenocarcinoma of the uterine cervix treated with radical hysterectomy and systematic lymphadenectomy. J Gynecol Oncol 24 222-228, 2013.

17. He L, Thomson JM, Hemann MT, et al: A microRNA polycistron as a potential human oncogene. Nature 435: 828-833, 2005

18. Zhang H, Li Y and Lai M: The microRNA network and tumor metastasis. Oncogene 29: 937-948, 2010.

19. Chen W, Cai F, Zhang B, Barekati Z and Zhong XY: The level of circulating miRNA-10b and miRNA-373 in detecting lymph node metastasis of breast cancer: potential biomarkers. Tumour Biol 34 455-462, 2013.

20. Xu Y, Sun J, Xu J, Li Q, Guo Y and Zhang Q: miR-21 is a promising novel biomarker for lymph node metastasis in patients with gastric cancer. Gastroenterol Res Pract 2012: 640168, 2012.

21. Wu WY, Xue XY, Chen ZJ, et al: Potentially predictive microRNAs of gastric cancer with metastasis to lymph node. World J Gastroenterol 17: 3645-3651,2011.

22. Zhao S, Yao D, Chen J and Ding N: Circulating miRNA-20a and miRNA-203 for screening lymph node metastasis in early stage cervical cancer. Genet Test Mol Biomarkers 17: 631-636, 2013.

23. Lund AH: miR-10 in development and cancer. Cell Death Differ 17: 209-214, 2010.

24. Ørom UA, Nielsen FC and Lund AH: MicroRNA-10a binds the 5'UTR of ribosomal protein mRNAs and enhances their translation. Mol Cell 30: 460-471, 2008.

25. Gaur A, Jewell DA, Liang Y, et al. Characterization of microRNA expression levels and their biological correlates in human cancer cell lines. Cancer Res 67: 2456-2468, 2007.

26. Varnholt H, Drebber U, Schulze F, et al. MicroRNA gene expression profile of hepatitis $\mathrm{C}$ virus-associated hepatocellular carcinoma. Hepatology 47: 1223-1232, 2008.
27. Volinia S, Calin GA, Liu CG, et al. A microRNA expression signature of human solid tumors defines cancer gene targets. Proc Natl Acad Sci USA 103: 2257-2261, 2006

28. Garzon R, Garofalo M, Martelli MP, et al: Distinctive microRNA signature of acute myeloid leukemia bearing cytoplasmic mutated nucleophosmin. Proc Natl Acad Sci USA 105: 3945-3950, 2008.

29. Kotelevets L, van Hengel J, Bruyneel E, Mareel M, van Roy F and Chastre E: The lipid phosphatase activity of PTEN is critical for stabilizing intercellular junctions and reverting invasiveness. J Cell Biol 155: 1129-1135, 2001.

30. Li J, Yen C, Liaw D, et al: PTEN, a putative protein tyrosine phosphatase gene mutated in human brain, breast, and prostate cancer. Science 275: 1943-1947, 1997.

31. Tamura M, Gu J, Matsumoto K, Aota S, Parsons R and Yamada KM: Inhibition of cell migration, spreading, and focal adhesions by tumor suppressor PTEN. Science 280: 1614-1617, 1998.

32. Carracedo A, Alimonti A and Pandolfi PP: PTEN level in tumor suppression: how much is too little? Cancer Res 71: 629-633, 2011.

33. Eijsink JJ, Noordhuis MG, ten Hoor KA, et al: The epidermal growth factor receptor pathway in relation to pelvic lymph node metastasis and survival in early-stage cervical cancer. Hum Pathol 41: 1735-1741, 2010

34. Lu D, Qian J, Yin X, Xiao Q, Wang C and Zeng Y: Expression of PTEN and survivin in cervical cancer: promising biological markers for early diagnosis and prognostic evaluation. $\mathrm{Br}$ J Biomed Sci 69: 143-146, 2012.

35. Zhu L, Loo WT and Louis WC: PTEN and VEGF: possible predictors for sentinel lymph node micro-metastasis in breast cancer. Biomed Pharmacother 61: 558-561, 2007.

36. Ettl T, Gosau M, Brockhoff G, et al: Predictors of cervical lymph node metastasis in salivary gland cancer. Head Neck 36: 517-523, 2014

37. Damsky WE, Curley DP, Santhanakrishnan M, et al: Beta-catenin signaling controls metastasis in Braf-activated Pten-deficient melanomas. Cancer Cell 20: 741-754, 2011.

38. Persad S, Troussard AA, McPhee TR, Mulholland DJ and Dedhar S: Tumor suppressor PTEN inhibits nuclear accumulation of beta-catenin and $\mathrm{T}$ cell/lymphoid enhancer factor 1-mediated transcriptional activation. J Cell Biol 153: 1161-1174, 2001.

39. Jamieson C, Sharma M and Henderson BR: Wnt signaling from membrane to nucleus: beta-catenin caught in a loop. Int J Biochem Cell Biol 44: 847-850, 2012.

40. Giangreco A, Lu L, Vickers C, et al: Beta-Catenin determines upper airway progenitor cell fate and preinvasive squamous lung cancer progression by modulating epithelial-mesenchymal transition. J Pathol 226: 575-587, 2012

41. Noordhuis MG, Fehrmann RS, Wisman GB et al: Involvement of the TGF-beta and beta-catenin pathways in pelvic lymph node metastasis in early-stage cervical cancer. Clin Cancer Res 17: 1317-1330, 2011.

42. Li XQ, Yang XL, Zhang G, et al: Nuclear $\beta$-catenin accumulation is associated with increased expression of Nanog protein and predicts poor prognosis of non-small cell lung cancer. J Transl Med 11: 114, 2013.

43. Ramachandran I, Thavathiru E, Ramalingam S, et al: Wnt inhibitory factor 1 induces apoptosis and inhibits cervical cancer growth, invasion and angiogenesis in vivo. Oncogene 31: 2725-2737, 2012

44. Heuberger J and Birchmeier W: Interplay of cadherin-mediated cell adhesion and canonical Wnt signaling. Cold Spring Harb Perspect Biol 2: a002915, 2010. 